\title{
Unscrambling English word order
}

\author{
Allan Ramsay \& Helen Seville \\ Centre for Computational Linguistics \\ UMIST, PO B Box 88, Manchester M60 1QD, England \\ allan/heleng@ccl.umist.ac .uk
}

\begin{abstract}
Wo propose a treatment of 'extraposition' which allows items to be assimilated directly even when they appcar far from their canonical positions. This treatment supports analyses of a number of phenomena which are otherwise hard to describe. The approach requires a generalisation of standard chart parsing techniques.
\end{abstract}

\section{Extraposition in English}

It is widely accepted that sentences such as

1 I saw the girl who your brother said he fancied.

2 The soup was OK, but the main course I thought was awful.

involve items ('who', 'the main course') being found far away from their normal positions (as the complement of 'fancied' and the subject of 'was awful'). It seems likely that the modifier's 'in the park' and 'with all my heart' in

3 In the park I met Arthur.

4 I believed with all my heart that she loved me.

are also 'out of position', since you would normally cxpect VP-modifying PI's of this kind to appear immediately to the right of the modified VP (so that the canonical versions of these sentences would have been 'I met Arthur in the park' and 'I believed that she loved me with all my heart.'). There are various reasons for moving things around in this way -moving 'who' to the left in (1) provides an easy way of picking out the boundary of the relative clause; moving 'the main course' and 'in the park' in (2) and (3) puts them into thematically/informationally more prominent positions; and moving the sentential complement 'that she loved me' to the right in (4) reduces the attachmont ambiguity that arises in the altermative form.

This is all well-known, and is treated in most grammatical framoworks by hallucinating an item in the canonical position, and then remembering that hallucination up to the point at which the out-ofplace item is encountered. Exactly how the hallucination is remembered varies from one framework to another, with unification grammars generally carrying information about it on a category-valued feature (usually called slash). The main problem with this approach is that it is difficult to control the sitnations in which 'traces' of this kind get proposed. (Johnson and Kay, 1994) suggest using 'sponsors' in order to license the introduction of traces, where a sponsor is some item of the required kind that has already been found, and which is hence potentially going to cancel with the trace.

If your parser works from left $\rightarrow$ right then this will work for items which have been left-shifted, but clearly it cannot work for right-shifted items, since the sponsor will not have been found at the time when it is noeded. Thus we camnot use a sponsor to justify hallucinating an S-comp for 'believed' in (4), or for the heavy-NP-shifts in

\section{He gave up his job.}

6 He built on that spot the most appallingly ugly house.

In any case, the notion that some item has becn left- or right-shifted fails to account for cases of 'intraposition':

\section{I believe Betty is a fool. \\ 8 Betty, I believe, is a fool.}

9 Betty is, I believe, a fool.

It is at least plausible that (8) and (9) are variants on (7). They're made out of the same words, they have the same truth conditions: the only trouble is that part of the sentence seems to be in the wrong place.

This is analogous to the situation in (2) and (3), where itcms were moved to the front to make them more prominent. It seems as though in the current case the words 'I belicve' have been shifted into the middle, and parenthesised, to make them less prominent. We will show how to deal with this by adapting 


\section{Charles danced with Eliza, but Diana he kissed.}

and when we say that the PP that results from saturating 'on' modifies a VP to its left we are referring to cases like (12), not to

\section{On the mat the cat sat.}

(or cven 'On the mat sat the cat.', where the expectation that the subject will appear to the left of the verb has also been violated.)

It seems as though we need the standard FRCP to cope with the canonical cases; the weakened FRCP' to cope with cases where the phrase occurs in some unexpected position; and something else to constrain the unexpected positions which are actually possible.

The constraints on what can be moved around take two forms. Firstly, we have say whether something can be moved at all, which we do by introducing a polar-valued feature called moved: items which appear away from their canonical positions are marked moved (right) or moved(left), depending on the direction in which they have been shifted. Arguments and targets which aren't allowed to move will be marked - moved by the item that subcategorises for them.

Sccondly, we have to specify where those items that can move are allowed to get to. We do this by using linear precedence rules (LP-rules), most of which place constraints on immediatc local subtrees. Thus we can say things like

$$
\begin{array}{r}
\{A, B, C\}:+w h @ A \&-w h @ B \\
\rightarrow \text { start@A } A<\text { start@B }
\end{array}
$$

to capture the fact that if $A$ and $B$ are local subtrees of $\mathrm{C}$, then if $A$ is WH-marked and $B$ is not then $A$ must precele $B$ ( $A$ 's start must be bofore $B$ 's). Note that the signature of the rule montions $C$, even though in this case the body does not.

The facts about extraposition are captured by rules which specify the circumstances under which a local subtree can (or must) be +moved. The key constraints for trees representing structures with verbal heads are as follow:

$$
\begin{aligned}
\{A, B, C\}: & -w h @ A \&-a u x @ C \& A=\text { subject@ } C \\
& \rightarrow-m o v e d @ A
\end{aligned}
$$

(if $A$ is the subject of $C$, where $A$ is not WH-marked and $C$ is not an auxiliary, then $A$ may not be moved)

$$
\begin{aligned}
& \{A, B, C\}: \operatorname{moved} @ A=r i g h t \\
& \rightarrow \exists X(X \in d t r s @ C \\
& \& \text { start@core@C }<\text { start@X) } \\
& \& \text { start@X } X<\operatorname{start} @ A)
\end{aligned}
$$

(if $A$ has been right-shifted, then $C$ had better have some other daughter $X$ between $C$ 's head and $A$.
The full rule says that $C$ must be heavier than $X$, where we take ' $C$ is heavicr than $X$ ' to mean that $C$ covers more words than $X$, so that this rule covers $(4),(5)$ and $(6))$.

There are a number of other such rules, of which the most complex relates to 'that'-clauses (denoted by tcomp). The description of such clauses comes in two parts, one to say that $-w h$ phrases may not be extracted and one to say that $+w h$ phrases must, be extracted.

(i) $\{A, B, C\}: C \in$ clause

$$
\begin{aligned}
& \&+\text { comp@C\& + compact } @ C \\
& \rightarrow-w h @ B
\end{aligned}
$$

(ii) $\{A, B, C\}: C \in$ clause

$$
\begin{aligned}
& \&+\text { comp@C\& - compact@C } \\
& \rightarrow+w h @ B
\end{aligned}
$$

The first part of this says that if combining $A$ and $B$ produces a 'that'-clause $C$, then if $C$ is +compact (so nothing has been extracted from it) then it had better be $-w h$ (in other words, nothing properly inside it can be $+w h$ ). If on the other hand $C$ is -compact then there must be something extracted from it, in which case the item which has been extracted must mark it as $+w h$. These rules cover the (un)acceptability of
15 I know that she loves me.
16 * I know me that she loves.
17 who I know that she loves.
18 * I know that who she loves.

\section{Intraposition}

The rules in Section 4 provide a reasonable account of simple extraposition (both left and right) from clauses. We now return to

\section{I believe Betty is a fool.}

8 Betty, I believe, is a fool.

9 Betty is, I believe, a fool.

Suppose we use FRCP', with no LP-rules, to analyse (8). We will get, among other things, the phrases and part phrases shown in Fig. 1 (the commas are treated as lexical items, so that 'Betty' starts at 0 , the first comma at 1 , and ' $I$ ' at 2 , and so on).

The first couple of steps are straightforward: ' $a$ fool' results from combining ' $a$ ' and 'fool'. It has no holes in it, its extreme start and end are the same as its compact start and end. Then 'is a fool' results from combining ' $i s$ ' and ' $a$ fool', and again all the pieces are in the right place, so the extreme start and end are the same as the compact start and end and the phrase is +compact.

At step 3, 'Betty' is integrated as the subject of 'is a fool'. The rosult starts at 0 , since that's where 


\begin{tabular}{|c|c|c|c|c|c|c|}
\hline & phrasc & start & cnd & xstart & xond & compact \\
\hline 1 & a fool & 6 & 8 & 6 & 8 & + \\
2 & is a fool & 5 & 8 & 5 & 8 & + \\
3 & Betty is a fool & 5 & 8 & 0 & 8 & - \\
4 & believe Betiy is a fool & 3 & 4 & 0 & 8 & - \\
5 & I believe Betty is a fool & 2 & 4 & 0 & 8 & - \\
6 & I believe Betty is a fool & 0 & 4 & 0 & 8 & - \\
7 & I believe Betty is a fool, & 0 & 8 & 0 & 8 & + \\
\hline
\end{tabular}

Figure 1: Analysis of (8)

'Betty' starts, and is -compact, since it does not include all the intervening words.

At 4 this -compact sentence becomes the complement of 'belicve'. The result is again - compact, since it fails to include the word ' $I$ ' or the two commas which appoar between its start and end points. The compact core is now the word 'believe', so the compact, start and end are 3 and 4 .

At 5, the VP 'believe Betty is a fool' combines with ' $I$ ' to produce 'I belicve Betty is a fool'. The two commas then combine with this phrase, marking it as being parenthetical and, when the second comma is included, finally marking it as + compact.

Similar structures would be created during the processing of (9), with the only difference being that 'is a fool' would be the first -compact phrase found. Apart from that the analysis of $(9)$ would be identical to the analysis of (8).

There are two problems with this approach to sentences of this kind: (i) because we obtain identical syntactic analyses of (7), (8) and (9), then any compositional semantics will assign all thee the same interpretation. This is not entirely wrong: I cannot fairly say any of these sentences unless I do believo that Betty is a fool. But it is also chearly not entirely right, since it misses the difference in emphasis. Wo will not discuss this any further locre. (ii) because wo are not applying the LP-rules, wo get rather a large number of analyses. Without LP-rules, we get, a singlo analysis of 'I believe Betty is a fool', having constructed 23 partial and complete edges. For 'Betty, $I$ believe, is a fool' we get three analyses (including the correct one) having constructed 101 edges. Most of these arise from the presence of the commas, since we have to allow for the possibility that each of these commas is either an opening or closing bracket, or a conjunction in a comma-separated list of conjuncts. Others arise from the fact that we have removed all the LP'rules, so that we are trcating English as having completely free word order. Case marking still provides some constraints on what can combine with what, so that in the current case ' $I$ ' is the only possible subject for 'believe' and 'Betty' is the only possible subject for 'is'. If we had been dealing with

\section{Betty, Fred believes, is a fool}

then wo would have had six analyses from 107 edges, with the new ones arising because we had assigned 'Fred' as the subject of 'is' and 'Betty' as the subject of 'believes'.

Clcarly we neod to reinstate the general LP-rules, whilst allowing for the cases we are interested in. These cases are characterised in two ways: (i) some word that requires a sentence lias occurred in a context where a 'split' sentence is available, and (ii) this word is adjacent to a parenthetical comma. The statement of this rule is rather long-winded, but the result is to provide a single analysis of (8) from 60 edges, and a single analysis of (9) from 70 edges.

\section{6 'more $X$ than $Y$ '}

Most cases of extraposition in English involve sontences, but there are a number of other phenomena where items seom to have beon shifted around. Consider for instance the following examples:

\section{George ate more than six peaches.}

21 Harriet ate more peaches than pears.

22 Ian ate more peaches than Julian.

In (20), 'more than six' looks like a complex cleterminer. How many peaches did George eat? More than six. The casiest way to analyse this is by assuming that 'more' subcategorises for a 'thanphrase'.

In (21) and (22), however, the than-phrase scems to have become disjointed. It still scems as though 'more' heads a complex determiner, since (22) would support the answer 'more than Julian' to the question 'How many peaches did Ian eat?' 3 .

We therefore introduce lexical entries for 'more' and 'than' which look roughly as follows:

\footnotetext{
"though (21) does not seem to support 'more than pears' as an answer to 'How many peaches did Harriet eat?'. 'The problem seems to be that $\mathrm{NP}^{3}$ complements to 'Than' are actually elliptical (see below), and it seems to be hasder to recover the ellipsed sentence 'More than she ate pear's' than to recover 'More than Julian ate peaches' or 'more than Julian ale'.
} 


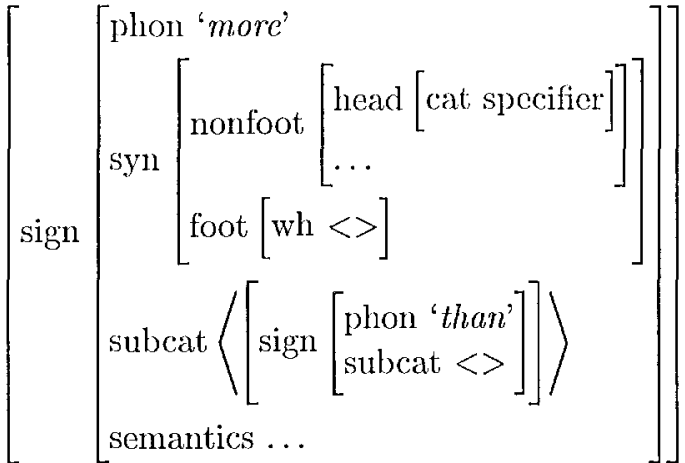

$$
\begin{aligned}
& {\left[\operatorname{sign}\left[\operatorname{syn}\left[\begin{array}{l}
\text { phon 'than' } \\
\text { nonfoot }\left[\begin{array}{l}
\text { head }[\text { cat than }] \\
\ldots
\end{array}\right] \\
\text { foot }[\text { wh }\langle\rangle] \\
\text { subcat }\langle\mathrm{X}\rangle \\
\text { semantics } \ldots
\end{array}\right]\right]\right.}
\end{aligned}
$$

The entry for 'more' says that it will make a specifier' if it finds a saturated phrase headed by 'than'. The entry for 'than' says that it will make a phrase of the required type so long as it finds some argument $X$. We know very little about $X$. In (20) it is a number, in (21) and (22) it appears to be an NP. In fact, as (Pulman, 1987) has shown, the best way to think about these cxamples is by regarding them as elliptical for the sentences

23 Harrict ate more peaches than she ate pears.

24 Ian ate more peaches than Julian ate peaches.

Other kinds of elliptical phrase are permitted, as in

25 Keith ate more peaches than Lucy did.

or cven

\section{Martha ate more ripe than unripe peaches. ${ }^{4}$}

We therefore allow arbitrary phrases as the argument to 'than'. All we need now are the LP-rules describing when arguments of 'than' should be extraposed. These simply say that if you are combining the determiner 'more' with a 'than'-phrase, then if the sole daughter of the 'than'-phrase is a number then it must not be shifted, and if it is not then it must be right-shifted.

(i) $\{A, B, C\}: A \in \operatorname{det} \& p h o n @ A=$ 'more' \& $\operatorname{cat} @ B=\operatorname{than} \& d \operatorname{trs}_{\mathrm{s}} @ B=\langle D\rangle$ $\&(D \in$ num or $D \in a d j)$

\footnotetext{
${ }^{4}$ Note that in this case the argument of 'than' is not displaced.
}

$$
\begin{aligned}
& \rightarrow \quad-m o v e d @ B
\end{aligned}
$$

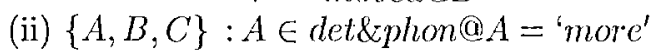

$$
\begin{aligned}
& \text { \& cat } @ B=\text { than } \\
& \& \operatorname{not}(\operatorname{dtr} s @ B=\langle D\rangle \\
& \&(D \in \text { num or } D \in \text { adj })) \\
& \rightarrow \text { moved@B } B=r i g h t
\end{aligned}
$$

With these LP-rules, we get appropriate structural analyses for $(20)-(25)$. We do not, however, currently have a treatment of ellipsis. We therefore camnot provide sensible semantic analyses of (21) and (22), since we cannot determine what sentences 'peaches' and 'Julian' are clliptical for (imagine, for instance, trying to decide whether 'Eagles eat more sparrows than crows' meant 'Eagles eat more sparrows than crows cats sparrows' or 'Eagles eat more sparrows than eagles eat crows').

If the structure of 'more peaches than pears' involves a displaced 'than'-phrase, then it seems very plausible that the same is true for

27 Nick wrote a more elegant program than Olive.

28 Peter wrote a more elegant program than that.

This is given further support by the acceptability of examples like

29 A program more elegant than that would be hard to find.

where the 'than'-phrase is adjacent to the modified adjective 'elegant' rather than to the noun 'program' which is modified by the whole phrase 'more elegant than that:

Frustratingly, it just does not seem possible to rouse the lexical entry above for 'more' to cope with these cases. In (20)-(25), 'more' made a determiner when supplied with an appropriate 'than'-phrase. For (27)- (29) it needs to make something which will combine with an adjective/adverb to produce an intensiffed version of the original. We therefore need the following entry:

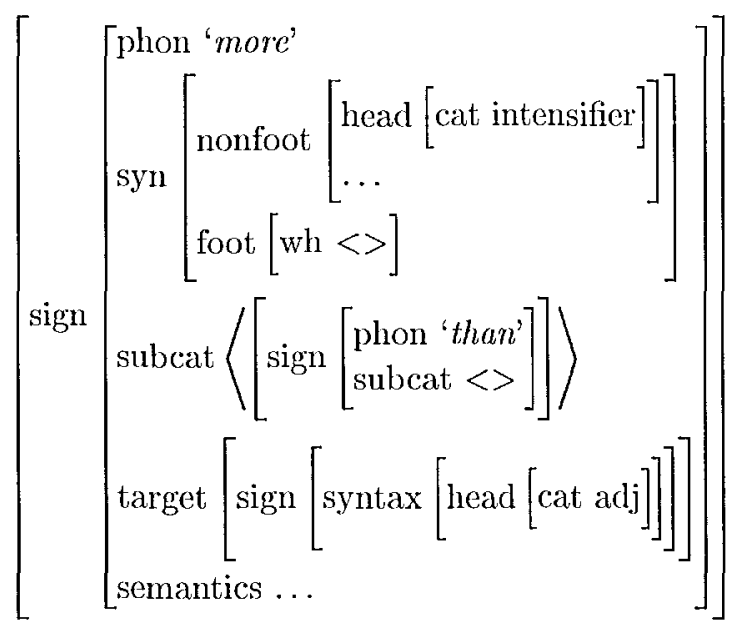


This needs a 'than'-phrase to saturate it, and once it is saturated it will combine with an adj (adjective or: adverb) to make a new adj. There are two questions to be answered: should such a complex adj appear to the left or right of its target, and should the 'than'phrase be extraposed or not?

(28) and (29) show that those questions are intimately comocted. If the 'than'-phrase is rightshifted, then the resulting modifier appears to the left of its target (28); if it is not, then the modifien appears to the right (29). This is exactly what is predicted by (Williams, 1981)'s suggestion that headfinal modifiers genorally appear to the loft of their. targets ('a quietly sleeping man') whereas non-headfimal onos appear to the right ('a man sleeping quictly'). All we need to do is to make right-shifting of the 'than'-phrase optional, and to invoke Williams' rule, using the compact cone of the modifier. Thus the compact modificr 'more elegant than that' from (20) is not head final, since the whole thing is compact but the head, 'elegant', is not, the last, word; the non-compact one 'more elegant . . than that' from (28) is head final, since this time 'elegant' is the last word in the compact core 'more elegant'. Hence 'more elegant than that' follows its target and 'more elegant ... than that' procedes it. No now IJP-rules are required, and no changes to the goneral rule for locating modifiers are required.

\section{Conclusions}

Wo have shown how retrieving displaced items directly, rather than positing a trace of some kind and then cancelling it against an appriate item when one turns up, can provide treatments of leftand right-cxtraposition which display the advantages that (Johnson and Kay, 1994) obtain for leftextraposition. This approach to extraposition can be extended to deal with 'intraposition' and to cases where items have been extracted from non-clausal items. In order to avoid overgencration, we needed to introduce a set of LP-rules which are applied as phrases are constructed in order to cnsure that items have not been shifted to macceptable positions. The extra computation required for checking the IAP-rules has no effect on the complexity of the parsing process, since they simply add a constant, (and fairly small) oxtra set of steps each time a ncw cdge is proposed. As a rough performance guide, the grammar generates five analyses for

30 He built on that site a more unatractive house than the one which he built in Greenwich.

on the basis of 237 edges (tho different global analyses arise from the attachment ambiguities for the various modificrs), and takes 4.1 seconds to do so (compiled Sicstus on a Pentium 350). This sentence contains a right-shifted NI, which itself contains a 'more ... than ...' construction and also a relative clause with a loft-shifted WH-pronoun, and hence could bo expected to cause problems for approaches using sponsors, while

\section{Betty, I belicve, is a fool.}

takes 0.27 seconds. The worst case complexity analysis for this kind of approach is fairly awful $\left(o\left(P^{2} \times 2^{2(N-1)}\right)\right.$ where $P^{\prime}$ is the number of unsaturated edges in tho initial chart and $N$ is the length of the sentence (Ramsay, in press)). In practice the LP-rules provide sufficicnt constraints on the generation of non-compact phrases for performance to bo gencrally acceptable on sentences of about twenty words.

\section{References}

M Johnson and M Kay. 1994. Parsing and emply nodes. Computational Linguistics, 20(2):289-300.

R M Kaplan. 1973. A gencral syntactic processor. In R. Rustin, editor, Natural language processing, pages 193-241, Now York. Algorithmics Press.

M Kay. 1973. The MINI system. In R. Rustin, editor, Natural Language Processing, pages 155 188, New York. Algorithmics Press.

C J Pollard and I A Sag. 1988. An Information Based Approach to Syntax and Semantics: Vol 1 Fundamentals. CSLI lecture notes 13, Chicago University Press, Chicago.

C J Pollard and I A Sag. 1994. Head-driven Phrase Structure Grammar. Chicago University Pross, Chicago.

S G Pulman. 1987. Events and VP-modifiers. In B.G.T. Lowden, editor, Proceedings of the Alvey Sponsored Workshop On Formal Semantics in Natural Language P'rocessing, Colchester. University of Esscx.

A M Ramsay. in pross. Parsing with cliscontinuous phrases. Natural Language Enginecring.

I A Sag and T Wasow. 1999. Syntactic theory: a formal introduction. CSLI, Stanford, Ca.

E Williams. 1981. On the notions 'lexically related' and 'hoad of a word'. Linguistic Inquiry, 12:254274.

M M Wood. 1993. Categorial Grammars. Routledge, London. 